\title{
Some properties of fuzzy quasi-metric space for FACS of fuzzy graph Type-3
}

Umilkeram Qasim Obaid ${ }^{\mathrm{a}, \mathrm{b}}$, Tahir Ahmad ${ }^{\mathrm{a}, \mathrm{c}, *}$

${ }^{a}$ Department of Mathematical Science, Faculty of Science, Universiti Teknologi Malaysia, 81310 Skudai, Johor, Malaysia

${ }^{b}$ Department of Mathematics., College of Science, AL-Mustansiriya University, Baghdad, Iraq.

${ }^{c}$ Centre for Sustainable NanoMaterials, Ibnu Sina Institute for Scientific and Industrial Research, Universiti Teknologi Malaysia, 81310 Skudai,

Johor,Malaysia

*Corresponding Author: tahir@ibnusina.utm.my

Article history :

Received 30 Oct 2014

Accepted 15 June 2015

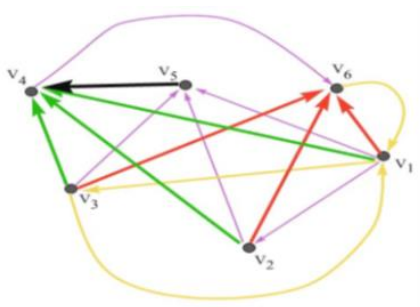

\section{ABSTRACT}

In this paper, we investigate some properties and characteristics of fuzzy quasi-metric space of Fuzzy Autocatalytic Set (FACS) of fuzzy graph Type-3. These properties are used to justify an aperiodicity of a graph.

Keywords: Fuzzy graph, Fuzzy Autocatalytic Set, Incineration process, Fuzzy quasi-metric space

(C) 2015 Penerbit UTM Press. All rights reserved http://dx.doi.org/10.11113/mjfas.v11n2.350

\section{INTRODUCTION}

A mathematical concept suggested by Zadeh in 1965 called fuzzy has been used to model the uncertainties of many real world applications. The participating of graph theory in fuzzy models has instigated the upgrowth of the fuzzy graph theory. An example in the application of fuzzy graph theory is in the modelling of clinical waste incineration process [1, 2]. The system was initially modelled using crisp graph as below.

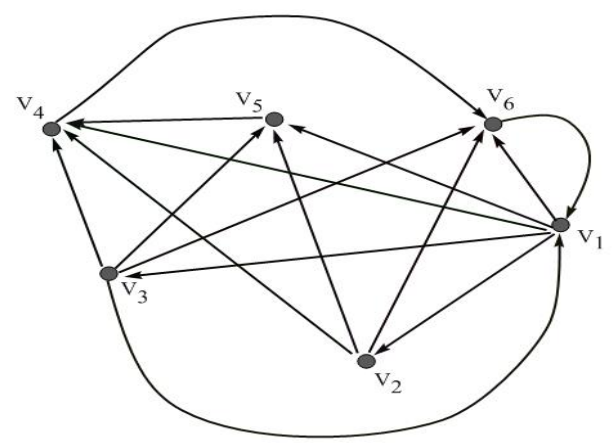

Figure.1 Crisp graph for the clinical waste incineration process where $\mathrm{V}_{1}$ : Waste, $\mathrm{V}_{2}$ : Fuel, $\mathrm{V}_{3}$ : Oxygen, $\mathrm{V}_{4}$ : Carbon Dioxide, $\mathrm{V}_{5}$ : Carbon Monoxide, $\mathrm{V}_{6}$ : Other gases including water
The model was found to be an Autocatalytic Set (ACS) [1]. However, the model is insufficient to explain the process [2]. Therefore, integration of fuzzy graph into the model has eventually created a new concept known as Fuzzy Autocatalytic Set (FACS) and shown to be a better in explaining the process $[1,2]$. Six important variables identified in the process are represented as nodes and the catalytic relationships are represented by fuzzy edges.

In this paper, we focus on the study of FACS of fuzzy graph Type-3 from a new perspective by using quasi-metric fuzziness. A new concept namely fuzzy quasi-metric space of FACS of fuzzy graph Type-3 was defined and implemented in the modelling of the incineration process [3]. A new notion of aperiodically convergent sequence in fuzzy quasi-metric space of FACS is offered and used to justify an aperiodic of a graph of FACS.

\section{FACS OF FUZZY GRAPH TYPE-3}

The major idea of the notion of FACS is the incorporation of fuzzy graph Type-3 to autocatalytic set. The definition of FACS is given as follows.

Definition 1: [1] Fuzzy autocatalytic set (FACS) is a subgraph where each of whose nodes has at least one incoming link with membership value $\mu\left(e_{i}\right) \in(0,1], \forall e_{i} \in$ E. 
A clinical waste incineration process in Malacca [2] was modelled using Definition 1 where the membership values for fuzzy edge connectivity for fuzzy graph are in the interval $(0,1]$. These values are revealed in the adjacency matrix of fuzzy graph defined as follows:

$$
C_{F_{i j}}=\left\{\begin{array}{cl}
0 & \text { if } i=j, \quad e_{i} \notin E \\
\mu\left(e_{i}\right) & \text { if } i \neq j
\end{array}\right.
$$

These values are determined through the chemical reactions taken place between six variables that play vital roles in the clinical waste incinerator, namely waste $\left(\mathrm{v}_{1}\right)$, fuel $\left(\mathrm{v}_{2}\right)$, oxygen $\left(\mathrm{v}_{3}\right)$, carbon dioxide $\left(\mathrm{v}_{4}\right)$, carbon monoxide $\left(\mathrm{v}_{5}\right)$ and other gases including water $\left(\mathrm{v}_{6}\right)$. From the explanation given in $[1,2]$ pertaining to the construction of FACS of fuzzy graph of Type-3 for the incineration process, the graph is represented as in Figure 2 and its adjacency matrix given in equation (1) is represented as in Figure 3.

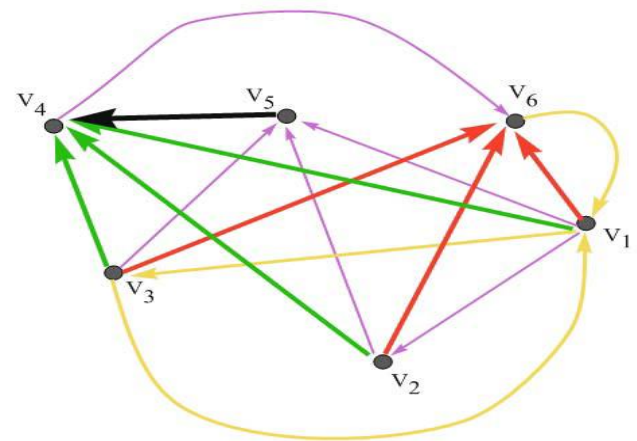

Figure.2 Fuzzy graph of Type-3 for the clinical waste incineration process

$C_{F_{i j}}=\left(\begin{array}{cccccc}0 & 0 & 0.06529 & 0 & 0 & 0.13401 \\ 0.00001 & 0 & 0 & 0 & 0 & 0 \\ 0.15615 & 0 & 0 & 0 & 0 & 0 \\ 0.51632 & 0.68004 & 0.63563 & 0 & 0.99999 & 0 \\ 0.00001 & 0.00001 & 0.00002 & 0 & 0 & 0 \\ 0.32752 & 0.31995 & 0.29906 & 0.00001 & 0 & 0\end{array}\right)$

Figure.3The adjacency matrix of FACS for the clinical waste incineration process [2]

The properties and characteristics of the graph deduced from adjacency matrix of FACS are explained in $[1,2]$, namely:

1. Strongly connected : each node in the graph has access to every other node.

2. Irreducible.

3. Aperiodic.

The above findings are benefit for the adjacency matrix construction of FACS for the clinical waste incineration process. However, the finding on irreducibility of FACS of fuzzy graph Type-3 was generalized using a new concept namely fuzzy quasi-metric space of FACS of fuzzy graph Type-3 as defined in [3]. We introduced the notion of a convergent sequence in fuzzy quasi-metric space of FACS and used it to justify an irreducibility of its graph [3]. In this paper, we will present the notion of an aperiodically convergent sequence to justify an aperiodicity of its graph.

\section{FUZZY QUASI-METRIC SPACE OF FACS OF FUZZY GRAPH TYPE-3}

In this section, some existing definitions and results that will be used in this work are reviewed. The definition of fuzzy quasi-metric space for FACS of fuzzy graph Type- 3 is given as below.

Definition 2: [3] Suppose $\mathrm{G}_{\mathrm{FT} 3}(\mathrm{~V}, \mathrm{E})$ be a no-loop Fuzzy Autocatalytic Set of fuzzy graph Type-3 and let a continuous t-norm is $a * b=a . b$ the usual multiplication. Let $M_{F T 3}$ be a fuzzy function defined by $M_{F T 3}\left(v_{i}, v_{j}, t\right)$ : $\mathrm{V} \times \mathrm{V} \times(0, \infty) \rightarrow(0,1]$

$$
M_{F T 3}\left(v_{i}, v_{j}, t\right)=\frac{t}{t+d_{F T 3}\left(v_{i}, v_{j}\right)}
$$

where $t$ is the number of edges in the $v_{i}-v_{j}$ fuzzy detour path $\mathrm{p}$ in FACS, $d_{F T 3}\left(v_{i}, v_{j}\right)$ is a fuzzy detour FT3-distance between two vertices $v_{i}$ and $v_{j}$ in FACS and $M_{\text {FT3 }}$ is satisfied the four conditions, for all $v_{i}, v_{j}, v_{k} \in \mathrm{V}$ and $t, s$ $>0$ :

1. $M_{F T 3}\left(v_{i}, v_{j}, t\right)>0$

2. $M_{F T 3}\left(v_{i}, v_{j}, t\right)=1$ if and only if $v_{i}=v_{j}$

3. $M_{F T 3}\left(v_{i}, v_{j}, t\right) * M_{F T 3}\left(v_{j}, v_{k}, s\right) \leq M_{F T 3}\left(v_{i}, v_{k}, t+s\right)$

4. $M_{F T 3}\left(v_{i}, v_{j},.\right):(0, \infty) \rightarrow(0,1]$ is continuous.

5

Then an ordered triple $\left(V, M_{F T 3}, \cdot\right)$ is said to be a fuzzy quasi-metric space of FACS and $\left(M_{F T 3}, \cdot\right)$ is called the fuzzy quasi-metric induced by $d_{F T 3}$ on FACS .

$M_{F T 3}\left(v_{i}, v_{j}, t\right)$ is denoted as $M_{F T 3}\left(v_{i}, v_{j}\right)$ and we will say that $M_{F T 3}$ is a fuzzy quasi-metric, or simply that $\left(V, M_{F T 3}\right)$ is a fuzzy quasi-metric space of FACS.

Next, the notion of a convergent sequence in a fuzzy quasi-metric space $\left(V, M_{F T 3}\right)$ of FACS is given and followed by a lemma and a theorem which is related to structural properties of the graph [3].

Definition 3: [3] A sequence $\left\{v_{k}\right\}$ in a fuzzy quasi-metric space $\left(V, M_{F T 3}\right)$ of FACS is converges to $v_{0}$ (i.e. $v_{k} \rightarrow v_{o}$ ) if there exists fuzzy detour path $\rho: v_{k}, v_{k-1}, \ldots, v_{0}$ of the maximum length $d_{F T 3}\left(v_{k}, v_{0}\right)$ such that $\lim _{k \rightarrow \infty} M_{F T 3}\left(v_{k}, v_{0}, t\right)=1$ for all $t>0$.

Lemma 1: [3] Let $\mathrm{G}_{\mathrm{FT} 3}(\mathrm{~V}, \mathrm{E})$ be a graph of FACS of fuzzy graph Type-3 and let $\left\{v_{k}\right\}$ be a sequence in a fuzzy quasimetric space $\left(V, M_{F T 3}\right)$ of FACS which converges to $v_{0}$. Then $\lim _{k \rightarrow \infty} M_{F T 3}\left(v_{k}, v_{0}, t\right)=1$ for all $t>0$ if and only if $\lim _{k \rightarrow \infty}\left\{d_{F T 3}\left(v_{k}, v_{0}\right): v_{k} \in \mathrm{V}\left(\mathrm{G}_{\mathrm{FT} 3}\right)-\left\{v_{0}\right\}\right\}=0$. 
Theorem 1: [3] Let the ordered pair ( $\left.V, M_{F T 3}\right)$ be a fuzzy quasi-metric space of FACS. Any graph of FACS of fuzzy graph Type-3 is strongly connected (irreducible graph) if and only if $\forall v_{o} \in V, \exists$ a sequence $\left\{v_{k}\right\}$ in a fuzzy quasimetric space $\left(V, M_{F T 3}\right)$ of FACS converges to $v_{o}$ (i.e. $v_{k}$ $\left.\rightarrow v_{o}\right)$.

\section{APERIODICITY OF FACS OF FUZZY GRAPH TYPE-3}

The study of aperiodicity for FACS of fuzzy graph Type-3, leads to the introduction of notion called aperiodic graph.

Definition 4: [4] A strongly connected graph is aperiodic if, for every node $i \in \mathrm{V}$, the greatest common divisor (gcd) of the length of all paths from $i$ to $i$ is equal to one.

In survey for FACS of fuzzy graph Type-3, the condition of aperiodicity (Definition 4) emerged due to the fact that a specific route is different to other route in the graph by its length of fuzzy detour path $\rho$ or by vertices that it passes through $\rho$. Aperiodicity means that a route is not periodic. This motivates the formation of the following definition that it is necessary for aperiodicity of FACS.

\section{Definition 5:}

A sequence $\left\{v_{i}\right\}$ in a fuzzy quasi-metric space $\left(V, M_{F T 3}\right)$ of FACS is aperiodically converges to $v_{i}\left(\right.$ e.i. $\left.v_{i} \stackrel{A P . C}{\longrightarrow} v_{i}\right)$ if there exists a fuzzy detour path that start and end at $v_{i}$ with path $\rho: v_{i}, v_{k}, \ldots, v_{j}, \ldots, v_{s}, v_{i}$ of the maximum length $d_{F T 3}\left(v_{i}, v_{i}\right)$ such that $\lim _{i \rightarrow \infty} M_{F T 3}\left(v_{i}, v_{i}, t\right)=1$ for all $t>0$ where $t$ is the number of edges in the $v_{i}-v_{i}$ fuzzy detour path $\rho$ in FACS.

Theorem 2: Let the ordered pair $\left(V, M_{F T 3}\right)$ be a fuzzy quasi-metric space of FACS. Any graph of FACS of fuzzy graph Type-3 is aperiodic if and only if $\forall v_{i} \in V, \exists$ a sequence of distinct vertices starting from $v_{i}$ and ending at $v_{i}$ in a fuzzy quasi-metric space $\left(V, M_{F T 3}\right)$ of FACS aperiodically converges to $v_{i}\left(\right.$ e.i. $\left.v_{i} \stackrel{A P . C}{\longrightarrow} v_{i}\right)$.

\section{Proof:}

Let $\mathrm{G}_{\mathrm{FT} 3}(\mathrm{~V}, \mathrm{E})$ be a graph of FACS of fuzzy graph Type-3 and is aperodic. It means that for every vertex $v_{i} \in V$, a path from $v_{i}$ to $v_{i}$ is not a multiple lengths of any existing routes in the graph. Hence, for every vertex $v_{i}$, there exists fuzzy detour path $\rho: v_{i}, v_{k}, \ldots, \ldots, v_{j}, \ldots, v_{s}, v_{i}$ with maximum length $d_{F T 3}\left(v_{i}, v_{i}\right)$. Now, it is left to show that $\lim _{i \rightarrow \infty} M_{F T 3}\left(v_{i}, v_{i}, t\right)=1$ for all $t>0$ and this is obtained by Lemma 1 . Therefore, by Definition $5, \exists$ a sequence starting from $v_{i}$ and ending at $v_{i}$ in a fuzzy quasi-metric space $\left(V, M_{F T 3}\right)$ of FACS aperiodically converges to $v_{i}$, $\forall v_{i} \in V$.
Conversely, suppose for every $v_{i} \in V, \exists$ a sequence of distinct vertices starting from $v_{i}$ and ending at $v_{i}$ in a fuzzy quasi-metric space ( $V, M_{F T 3}$ ) of FACS aperiodically converges to $v_{i}$. This means that there exists fuzzy detour path $\rho: v_{i}, v_{k}, \ldots, v_{j}, \ldots, v_{s}, v_{i}$ of the maximum length $d_{F T 3}\left(v_{i}, v_{i}\right)$ such that $\lim _{i \rightarrow \infty} M_{F T 3}\left(v_{i}, v_{i}, t\right)=1$ for all $t>$ 0 . Hence, the graph of FACS of fuzzy graph Type- 3 is aperiodic.

\section{CONCLUSION}

A new concept namely an aperiodically convergent sequence in a fuzzy quasi-metric space of FACS of fuzzy graph Type-3 is presented and used to justify an aperiodicity of a graph.

\section{ACKNOWLEDGEMENT}

This research is supported by Research University Grant (06H16) awarded by Universiti Teknologi Malaysia (UTM). The researchers are thankful to its financial support.

\section{REFERENCES}

[1] Ahmad, T.; Baharum, S.; Arshad, K.A. Modeling a clinical incineration process using fuzzy autocatalytic set, Journal of Mathematical Chemistry, 47(2010) 1263-1273.

[2] Baharum, S.; Ahmad, T.;Yusof, M.R. Relationship between Fuzzy Edge Connectivity and the Variables in Clinical Waste Incineration Process, MATEMATIKA, 25(2009) 31-38.

[3] Obaid, U.Q.; Ahmad, T. Fuzzy quasi-metric space of Fuzzy Autocatalytic Set (FACS) of fuzzy graph Type-3, Life Science Journal,11(2014) 738-746.

[4] Ninove, L. Dominant Vectors of Nonnegative Matrices: Application to Information Extraction in Large Graphs. Doctor Philosophy. Universite Chatolique De Louvain, France, 2008. 